\title{
Impact of hypertension on cognitive performance in individuals with high level of education
}

\author{
Henrique CS Muela $^{1,2}$, Valeria A Costa-Hong' ${ }^{1}$, Mônica S Yassuda ${ }^{3}$, Natália C Moraes ${ }^{3}$, Claudia M Memória ${ }^{3}$, Thiago A Macedo ${ }^{1}$, Michel F \\ Machado $^{3}$, Edson Bor-Seng-Shu ${ }^{3}$, Ayrton R Massaro ${ }^{3}$, Ricardo Nitrini ${ }^{3}$ and Luiz A Bortolotto ${ }^{1}$ \\ ${ }^{1}$ Heart Institute (INCOR), University of São Paulo Medical School, São Paulo, Brazil \\ ${ }^{2}$ Department of Physiology, Faculty of Medicine, Agostinho Neto University, Luanda, Angola \\ ${ }^{3}$ Department of Neurology, University of São Paulo Medical School, São Paulo, Brazil
}

\begin{abstract}
Education is considered to provide a cognitive and neurological reserve through neuronal changes or increased efficacy of processing networks. The hypothesis of cognitive reserve asserts that older individuals with greater experiential resources exhibit better cognitive functioning and are able to tolerate higher levels of brain pathology before displaying clinical symptoms. Hypertension is linked to cognitive impairment. We evaluated the impact of hypertension on cognitive performance in hypertensive patients with high level of education. In a cross-sectional study 163 patients (61 normotensive and 102 hypertensive) with 9 or higher schooling years were comparatively evaluated. Hypertension was defined as blood pressure (BP) levels $\geq 140 / 90 \mathrm{mmHg}$ or use of antihypertensive drugs. The mini-mental state examination (MMSE), Montreal Cognitive Assessment (MoCA), and a validated comprehensive battery of neuropsychological tests that assessed 6 main cognitive domains were used to determine cognitive function. Hypertension group had higher weigh, BMI and lower education level and family income. Hypertension group performed worse either on MMSE ( $27.7 \pm 1.9$ vs $28.3 \pm 1.7$, $\mathrm{p}=0.049)$ or MoCA $(25.2 \pm 2.6$ vs $26.1 \pm 2.5$, $\mathrm{p}=0.022)$; similar poorer performance in hypertensive group were also seen at different domains on neuropsychological evaluation. We concluded that even in those with high level of education, patients with hypertension have poorer cognitive performance compared to control pairs.
\end{abstract}

\section{Introduction}

Education is considered to provide a cognitive and neurological reserve through neuronal changes or increased efficacy of processing networks [1]. The "reserve" hypothesis suggests that education should affect the clinical expression of Alzheimer's disease (AD). The concept of cognitive reserve has been proposed to account for the disjunction between the degree of brain damage or pathology and its clinical manifestations [2]. Twenty-five percent of elders whose neuropsychological testing is unimpaired prior to death meet full pathologic criteria for $\mathrm{AD}$, [3] suggesting that this degree of pathology does not invariably result in clinical dementia. Educational and occupational exposure and leisure activities are considered that as related with a reduced risk of developing dementia [2]. Neuropathologic correlations support this theory showing that individuals with greater cognitive reserve, as reflected in years of education, are better able to cope with $\mathrm{AD}$ brain pathology without observable cognitive deficits [4].

The hypothesis of cognitive reserve asserts that older individuals with greater experiential resources exhibit better cognitive functioning and are able to tolerate higher levels of brain pathology before displaying clinical symptoms [5]. One of the most well-established proxy measures of reserve capacity in the elderly is educational attainment, which is thought to reflect more effective use of brain networks or cognitive paradigms [2]. In line with the hypothesis of cognitive reserve, many studies in both North America and Europe have suggested that educational attainment is associated with better cognitive performance and reduced risk for cognitive impairment and dementia in late life [6,7].

Results with respect to whether educational attainment moderates the trajectory of age-related cognitive decline have been mixed [8].
Several studies reported that educational attainment attenuates cognitive decline in samples of non-demented, older adults $[9,10]$. Such results support an active cognitive reserve hypothesis in which education promotes more efficient cognitive processing and use of brain networks, which results in smaller cognitive declines in the face of neuropathology, effectively slowing the process of age-related cognitive decline. [11].

The importance of educational level on neuropsychological tests performance has been related in several kinds of abilities, such as memory, language, problems solving and constructional praxis as well as motor and calculation abilities. For the interpretation of results in cognitive tasks, education must be taken up. However, differentiation of what is an educational variable and what is a cognitive deficit is a hard process. If neuropsychological evaluation does not take enough care, it can suggest a neurological pathology where there are only educational deprivation. [12].

Both hypertension (HTN) and low education level have been implicated with poorer cognitive performance [13]. However, whether the high level of education may play the same "protective role" on the cognitive function in presence of hypertension is unknown.

Correspondence to: Henrique CS Muela. Department of Physiology, Faculty of Medicine, Agostinho Neto University, Luanda, Angola. POX: 116, Tel: +244 925141398. E-mail: henrimuela@hotmail.com

Key words: hypertension, cognitive impairment, education, high school

Received: July 23, 2017; Accepted: August 05, 2017; Published: August 08 2017 
Thus, in the present study we aimed to evaluate the impact of hypertension on cognitive function in hypertensive patients with high level of education.

\section{Methods}

In a cross-sectional study 163 patients (61 normotensive and 102 hypertensive) were comparatively evaluated. Hypertension was defined as blood pressure (BP) levels $\geq 140 / 90 \mathrm{mmHg}$ or use of antihypertensive drugs. Patients with hypertension were recruited among those enrolled and followed regularly in the hypertension unit of the Heart Institute of the University of São Paulo Medical School. The normotension group participants were recruited among patients without cardiovascular (CV) disease followed yearly at the Heart Institute as part of a protocol for cardiovascular assessment. [14]

Patients with the following conditions were excluded: 8 schooling years or less, age less than 18 years old, overt cerebrovascular disease (previous stroke or transient ischemic attack), diabetes mellitus, smoking, arrhythmias, heart failure with left ventricular dysfunction and known neurodegenerative or psychiatric disease. Educational level was assessed by the number of completed schooling years.

The local ethics committee approved the protocol, and all participants gave written, informed consent.

\section{Blood pressure measurement}

Brachial systolic and diastolic blood pressure was assessed with an Omron automatic device, HEM-705 CP model, in the right upper arm, with the subject seated, after resting for 5 minutes following the recommendations of the VII Brazilian hypertension guidelines. [15] A mean of 3 measurements with a 1-minute interval between was calculated and used to determine systolic and diastolic blood pressure in each patient.

Patients with hypertension were divided into 2 groups, according to BP levels or medication use (HTN-1: BP 140-159/90-99 mm Hg or BP under control with 1 or 2 antihypertensive drugs; HTN-2: BP $\geq 160 / 100$ $\mathrm{mm} \mathrm{Hg}$ or BP under control with $\geq 3$ drugs). Controlled HTN was defined as BP levels $<140 / 90 \mathrm{mmHg}$ with the use of antihypertensive drugs.

\section{Cognitive function evaluation}

The mini-mental state examination (MMSE), Montreal Cognitive Assessment (MoCA), and a validated comprehensive battery of neuropsychological tests that assessed 6 main cognitive domains were used to determine cognitive function.

There is a wide variety of screening tests, each with different sensitivity and specificity for particular brain functional deficits. Cognitive screening instruments should ensure brief application, ease of use, and low cost, and able to be used to rule out pathological cognitive changes.

The Mini-Mental State Examination (MMSE) is the cognitive screening test frequently used in the clinical setting, but in stages where cognitive impairment is close to the changes of aging, scores on screening tests may be erroneously classified as within the normal range. In order to contemplate this gap, Nasreddine et al. [16] proposed the Montreal Cognitive Assessment (MoCA), a specific screening test for mild cognitive impairment (MCI) detection. This new assessment test incorporates subtests that assess executive functions and processing speed that are often compromised in vascular cognitive deficits.
However, the limitations of screening protocols in MCI detection point to the need for a detailed assessment of cognitive functions. The more detailed neuropsychological evaluation (NPE) has its importance when the clinical picture is ambiguous or complex, providing an early and reliable differentiation between normal aging and health conditions that involve cognitive impairment. It is an exam that evaluates brain functioning and may be useful in defining the nature and severity of behavioral and emotional problems resulting from brain injury or dysfunction [17].

\section{Mini Mental State Examination (MMSE)}

The MMSE is a commonly used 30-point scale for assessing cognitive function in the areas of orientation, registration, attention and calculation, recall, language, and praxis. MMSE administration was performed according to existing standards [18]. On the MMSE, the score of $\leq 23$ is usually an accepted cutoff indicating the presence of cognitive impairment. However, many authors have recommended the adjustment to different cutoffs, considering the level of education and not a limited cutoff score. The following cutoff scores were used to identify abnormal cognition in this study: $\leq 23$ for those with 9-11 years of education, and $\leq 25$ for those with $\geq 12$ years of education [19].

\section{Montreal Cognitive Assessment (MoCA)}

The MoCA was designed as a rapid screening instrument to identify mild cognitive impairment (MCI) [19]. It assesses different cognitive domains: attention and concentration, executive functions, memory, language, visuoconstructional skills, conceptual thinking, calculations, and orientation. The total possible score is 30 points and a score of 26 or above is considered normal. A previous validation study in Brazil suggested 25 points as the ideal cutoff for MCI identification [20]. To counterbalance the effect of lower education 1 point was added to the final score of those individuals with less than 12 years of education.

\section{Neuropsychological Evaluation (NPE)}

Procedures and descriptions of the neuropsychological tests used have been published elsewhere. [21-29]. The neuropsychological test battery included the Boston Naming Test (BNT) [21], Rey Auditory Verbal Learning Test (RAVLT5 - sum of 5 recall trials of 15 words, RAVLT6 - immediate recall after inference and RAVLT7 - delayed recall after 30 minutes) [25], the Rey-Osterrieth Complex Figure Test copy and delayed recall (REY-C and REY-30) [26], Semantic Verbal Fluency animal category (VF) [22], Phonological Verbal Fluency (FAS), [24] Forward and Backward Digit Span Test (FDST and BDST) [29], Trail Making Test part A and B (TMT-A and TMT-B) [23], Clock Drawing Test (CDT) [27], and Digit Symbols Substitution Test (DSST) [28].

We computed scores for global cognition (mean $\mathrm{z}$ score of the BNT, RAVLT5, RAVLT6, RAVLT7, REY-C, REY-30, VF, FAS, FDST, BDST, TMT-A, TMT-B, CDT and the DSST), language (BNT), memory (mean $z$ score of the RAVLT5, RAVLT6, RAVLT7 and REY30), executive functioning (mean $\mathrm{z}$ score of the VF, FAS, BDST and TMT-B), visuospatial abilities (mean $z$ score of the REY-C and CDT), attention (mean $\mathrm{z}$ score of the FDST and TMT-A) and processing speed (DSST).

$\mathrm{Z}$ scores were calculated using people without hypertension (control) as the reference group. Participants were considered to have cognitive impairment if they had scores below $-1.5 \mathrm{SD}$, on one or more cognitive domains. 


\section{Statistical analysis}

Data were analyzed with SPSS for Windows 21.0. The distribution of the data was determined using the Kolmogorov-Smirnov test. Continuous variables were presented as mean and standard deviation and analyzed by the independent samples $t$ test; categorical data were presented as percentages and analyzed by Mann Whitney test. Pearson or Spearman coefficients were used for bivariate correlations in hypertensive group wherever suitable. In multivariable regression analyses, all variables with $\mathrm{p}<0.1$ in unadjusted analysis were selected for multivariable analysis. In the models, we included cognitive variables as dependent variables and clinical parameters as independent viariables. Statistical significance was set at $5 \%$.

To account for group differences in education, this variable was included as a covariate in the models. To the best of our knowledge, variables such as weight, height, BMI, marital status and monthly income do not have significant impact on cognitive functions, so we did not use them as covariates in the models with cognitive performance as dependent variables.

\section{Results}

There were enrolled 163 patients (61 normotensive and 102 hypertensive) according to our exclusion criteria. Baseline characteristics of the study sample groups are described in Table 1. Hypertension group had higher weigh, BMI and lower education level and family income. Clinical parameters were almost similar except on SBP and DBP.

Cognitive performance on different tests is presented in Table 2. Hypertension group performed worse than the control group in almost cognitive tests on different domains, except memory domain. However, after educational level correction only the following variables remained statistically different: MoCA (25.2 vs. 26.1, $\mathrm{p}=0.039)$, Backward digit span $(-0.30$ vs. $0.14, \mathrm{p}=0.014)$, Rey-C $(-0.16$ vs. $0.30, \mathrm{p}=0.002)$, TMT-A ( -0.06 vs. $0.23, \mathrm{p}=0.006)$, Digit symbols substitution test $(-0.23$ vs. $0.16, \mathrm{p}=0.021)$.

Table 1. General characteristics of the study participants

\begin{tabular}{|l|c|c|c|}
\hline & Normotension & Hypertension & \\
\hline Variable - mean \pm SD & $\mathbf{- 6 1}$ & $\mathbf{- 1 0 2}$ & P value \\
\hline Age, years, mean \pm SD & $51.6 \pm 13.5$ & $49.7 \pm 12.1$ & 0.347 \\
\hline Sex, Male, No (\%) & $30(49.2)$ & $43(42.2)$ & 0.384 \\
\hline Race, White, No (\%) & $39(63.9)$ & $70(68.6)$ & 0.705 \\
\hline Married, No (\%) & $27(44.3)$ & $58(56.9)$ & 0.045 \\
\hline Weight $(\mathrm{kg})$, mean \pm SD & $74.6 \pm 17.2$ & $80.8 \pm 15.3$ & 0.019 \\
\hline Height $(\mathrm{m})$, mean \pm SD & $1.7 \pm 0.1$ & $1.7 \pm 0.1$ & 0.392 \\
\hline BMI $\left(\mathrm{kg} / \mathrm{m}^{2}\right)$ mean \pm SD & $26.5 \pm 4.2$ & $29.5 \pm 5.3$ & $<0.001$ \\
\hline Education(yrs) mean \pm SD & $14.1 \pm 2.8$ & $13.1 \pm 2.9$ & 0.029 \\
\hline Monthly income- \\
mean,range* & $3000(730-20.000)$ & $2000(600-20.000)$ & 0.001 \\
\hline Clinical parameters & & & \\
\hline SBP, mmHg, mean \pm SD & $122.0 \pm 8.5$ & $140.8 \pm 22.1$ & $<0.001$ \\
\hline DBP, mmHg, mean \pm SD & $76.2 \pm 7.1$ & $86.7 \pm 13.2$ & $<0.001$ \\
\hline Glycemia, mean \pm SD & $96.2 \pm 11.5$ & $100.1 \pm 10.0$ & 0.138 \\
\hline Total cholesterol, mean \pm SD & $211.1 \pm 46.7$ & $199.9 \pm 46.1$ & 0.365 \\
\hline HDL cholesterol, mean \pm SD & $57.6 \pm 16.5$ & $49.1 \pm 14.2$ & 0.032 \\
\hline LDL cholesterol, mean \pm SD & $129.6 \pm 40.0$ & $123.0 \pm 43.0$ & 0.63 \\
\hline Triglycerides, mean \pm SD & $130.9 \pm 72.9$ & $136.8 \pm 74.6$ & 0.766 \\
\hline Creatinine, mean \pm SD & $1.0 \pm 0.8$ & $1.0 \pm 0.2$ & 0.397 \\
\hline GFR, mean \pm SD & $85.2 \pm 17.9$ & $79.8 \pm 18.3$ & 0.263 \\
\hline
\end{tabular}

*1USD=3.6 Reais; BMI, body mass index; DBP, diastolic blood pressure; GRF, glomerular filtration rate; HDL, high density lipoprotein; LDL: low density lipoprotein; SD: standard deviation.
Cognitive performance on neuropsychological tests is presented in Table 3. Similarly, hypertension group had worse cognitive performance on different cognitive domains when compared to control group.

Bivariate correlation between cognitive and clinical variable in hypertensive patients is presented in Table 4. Age, hypertension stage and systolic blood pressure negatively correlated with cognitive function with either MMSE, MoCA or NPE on different domains; on the other hand, education level had positive correlation with cognitive tests on different domains. In the multivariate analysis (Table 5), age, education level and HTN stage were the variables that mostly predicted the cognitive impairment on different domains. The odds ratio of cognitive impairment on different tests in the patients with hypertension was respectively: MMSE (OR: 1.08, 95\%CI: 0.35-3.40); MoCA (OR: 1.54, 95\%CI: 0.73-3.24) and NPE (OR: 2.03, 95\%CI: 0.90-4.52).

Table 2. Cognitive performance according to the study participants

\begin{tabular}{|c|c|c|c|}
\hline \multirow{2}{*}{$\begin{array}{c}\text { Variable - mean } \\
\pm \text { SD }\end{array}$} & Normotension & Hypertension & P value \\
\hline MMSE & $28.3 \pm 1.7$ & $-\mathbf{- 1 0 2}$ & 0.049 \\
\hline MoCA & $26.1 \pm 2.5$ & $25.7 \pm 1.9$ & 0.022 \\
\hline \multicolumn{4}{|c|}{ Language } \\
\hline BNT & $0.09 \pm 0.89$ & $-0.16 \pm 1.09$ & 0.132 \\
\hline \multicolumn{5}{|c|}{ Memory } \\
\hline RAVLT5 (sum of 5 \\
trials)
\end{tabular}

Note: BNT, Boston naming test; $\mathrm{CDT}=$ clock drawing test; MMSE, mini mental state examination; MoCA, Montreal Cognitive Assessment; RAVLT, Rey Auditory Verbal Test Rey-C, Rey Osterrieth complex figure copy; Rey-30, Rey Osterrieth complex figure recall after 30 minutes; TMT, Trial Making Test part A and A. All analyses were adjusted to the education level (years of education).

Table 3. NPE Compound scores in specific cognitive domains according to blood pressure level

\begin{tabular}{|c|c|c|c|}
\hline \multirow{2}{*}{ Cognitive Domain - mean (SD) } & Normotension & Hypertension & \multirow{2}{*}{ p vale } \\
\cline { 2 - 3 } & $\mathbf{- 6 1}$ & $\mathbf{- 1 0 2}$ & \\
\hline Global Cognitive function $^{1}$ & $0.14 \pm 0.49$ & $-0.15 \pm 0.54$ & 0.001 \\
\hline Language & $0.09 \pm 0.89$ & $-0.16 \pm 1.09$ & 0.132 \\
\hline Memory & $0.14 \pm 0.70$ & $-0.14 \pm 0.96$ & 0.054 \\
\hline Executive function & $0.12 \pm 0.72$ & $-0.21 \pm 0.61$ & 0.002 \\
\hline Visuospatial abilities & $0.18 \pm 0.60$ & $-0.09 \pm 0.69$ & 0.012 \\
\hline Attention & $0.14 \pm 0.61$ & $-0.05 \pm 0.61$ & 0.051 \\
\hline Processing Speed & $0.16 \pm 0.90$ & $-0.23 \pm 0.84$ & 0.007 \\
\hline
\end{tabular}

${ }^{1}$ Global cognitive function was calculated as the mean z score of all neuropsychological tests. All analyses were adjusted to the education level (years of education). 
Table 4. Unadjusted correlation coefficients between clinical variables and cognitive tests in the hypertensive patients*

\begin{tabular}{|c|c|c|c|c|c|c|c|c|c|c|}
\hline \multirow[t]{2}{*}{ Variable } & \multicolumn{2}{|c|}{ Age } & \multicolumn{2}{|c|}{ Education } & \multicolumn{2}{|c|}{ HTN stage } & \multicolumn{2}{|c|}{ SBP } & \multicolumn{2}{|c|}{ DBP } \\
\hline & $\mathrm{r}$ & $\mathrm{p}$ & $\mathrm{r}$ & $\mathrm{p}$ & $\mathrm{r}$ & $\mathrm{p}$ & $\mathrm{r}$ & $\mathrm{p}$ & $\mathrm{r}$ & $\mathrm{p}$ \\
\hline MMSE & 0.037 & 0.642 & 0.222 & 0.004 & -0.136 & 0.084 & -0.075 & 0.34 & -0.021 & 0.789 \\
\hline $\mathrm{MoCA}$ & -0.076 & 0.338 & 0.119 & 0.129 & -0.171 & 0.029 & -0.08 & 0.308 & -0.018 & 0.819 \\
\hline GCF & -0.3 & $<0.001$ & 0.372 & $<0.001$ & -0.277 & $<0.001$ & -0.153 & 0.052 & -0.083 & 0.294 \\
\hline Language & -0.013 & 0.864 & 0.272 & $<0.001$ & -0.11 & 0.13 & -0.105 & 0.181 & -0.042 & 0.596 \\
\hline Memory & -0.304 & $<0.001$ & 0.201 & 0.01 & -0.166 & 0.035 & -0.098 & 0.214 & -0.072 & 0.359 \\
\hline Executive function & -0.156 & 0.046 & 0.414 & $<0.001$ & -0.268 & 0.001 & -0.112 & 0.155 & -0.039 & 0.618 \\
\hline Visuospatial ability & -0.063 & 0.427 & 0.13 & 0.098 & -0.187 & 0.017 & -0.063 & 0.425 & -0.043 & 0.584 \\
\hline Attention & -0.276 & $<0.001$ & 0.286 & $<0.001$ & -0.182 & 0.02 & -0.158 & 0.044 & -0.088 & 0.263 \\
\hline Processing speed & -0.396 & $<0.001$ & 0.22 & 0.005 & -0.223 & 0.004 & -0.142 & 0.071 & -0.066 & 0.403 \\
\hline
\end{tabular}

$\mathrm{BP}=$ blood pressure; $\mathrm{DBP}=$ diastolic blood pressure; $\mathrm{HTN}=$ hypertension; $\mathrm{GCF}=$ global cognition function; $\mathrm{SBP}=$ systolic blood pressure. $\mathrm{MMSE}=$ mini mental state examination; MoCA=Montreal cognitive assessment. *Pearson or Spearman coefficients were used for bivariate correlations wherever suitable.

Table 5. Multivariable analysis between clinical parameters as independent variables and cognitive tests as dependent variables in hypertensive patients.*

\begin{tabular}{|c|c|c|c|}
\hline Variable & & Parameter & \\
\hline & B & $95 \%$ CI for B & p \\
\hline & & MMSE & \\
\hline \multirow[t]{2}{*}{ Years of education } & 0.142 & $0.045,0.249$ & 0.004 \\
\hline & \multicolumn{3}{|c|}{ Global Cognitive Function } \\
\hline Age & -0.014 & $-0.019,-0.08$ & $<0.001$ \\
\hline HTN stage & -0.145 & $-0.226,-0.064$ & 0.001 \\
\hline \multirow[t]{2}{*}{ Years of education } & 0.06 & $0.034,0.085$ & $<0.001$ \\
\hline & & Memory & \\
\hline Age & -0.022 & $-0.032,-0.012$ & $<0.001$ \\
\hline HTN stage & -0.158 & $-0.301,-0.016$ & 0.03 \\
\hline \multirow[t]{2}{*}{ Years of education } & 0.05 & $0.005,0.094$ & 0.029 \\
\hline & \multicolumn{3}{|c|}{ Executive function } \\
\hline Age & -0.009 & $-0.017,-0.002$ & 0.014 \\
\hline HTN stage & -0.156 & $-0.260,-0.052$ & 0.004 \\
\hline \multirow[t]{2}{*}{ Years of education } & 0.086 & $0.053,0.118$ & $<0.001$ \\
\hline & \multicolumn{3}{|c|}{ Visuospatial abilities } \\
\hline \multirow[t]{2}{*}{ HTN stage } & -0.138 & $-0.250,-0.025$ & 0.017 \\
\hline & & Attention & \\
\hline Age & -0.014 & $-0.021,-0.007$ & $<0.001$ \\
\hline HTN stage & -0.108 & $-0.207,-0.010$ & 0.005 \\
\hline \multirow[t]{2}{*}{ Years of education } & 0.053 & $0.022,0.084$ & 0.001 \\
\hline & \multicolumn{3}{|c|}{ Processing speed } \\
\hline Age & -0.029 & $-0.038,-0.020$ & $<0.001$ \\
\hline HTN stage & -0.222 & $-0.356,0.088$ & 0.001 \\
\hline Years of education & 0.051 & $0.009,0.093$ & 0.017 \\
\hline
\end{tabular}

Note: B indicates unstandardized model coefficients that indicate how much the dependent variable varies with an independent variable when all other independent variables are held constant. Consider the effect of years of education in this example. The unstandardized coefficient, $\mathrm{B}_{1}$, for MMSE is equal to 0.142 . This means that for each 1year increase in education, there is an increase in MMSE of 0.142 . CI, confidence interval. * To run the multivariable analysis models, for each dependent variable (cognitive) all independen variables (clinical) with $\mathrm{p}<0.1$ in the bivariate analysis were selected. To be included in the multiviable models each dependent variabble should have at least two indepent variables with $\mathrm{p}<0.1$ in the bivariate correlation.

\section{Discussion}

The main result of this study is that even in individuals with high level of education, patients with hypertension perform worse on cognitive tests suggesting a deleterious effect of hypertension even in individuals where education is believed to have a protective effect from cognitive decline. Age, HTN stage and education level were the variables that mainly predicted the cognitive impairment.

Although the relationship between education and cognitive status is well-known, evidence regarding whether education moderates the trajectory of cognitive change in late life is conflicting. Early studies suggested that higher levels of education attenuate cognitive decline [30]. However, more recent studies using improved longitudinal methods have not found that education moderates cognitive decline. In the study where were analyzed data from 1,023 participants in the Victoria Longitudinal Study to examine the effects of education on composite scores reflecting verbal processing speed, working memory, verbal fluency, and verbal episodic memory education was not related to rates of change over time for any cognitive domain. [6].

Multiple studies have indicated that educational attainment modifies the association between a direct measure of brain pathology and neuropsychological test performance [32,33]. Such findings have led some researchers to consider education to be the key protective factor against dementia [34]. Valenzuela and Sachdev demonstrated that individuals with a high level of education had a $47 \%$ decrease in risk for dementia compared to those with a lower level educational attainment [35]. A recent study has confirmed that education up to year 12 has a dose-related effect on reducing risk of dementia with advancing age, irrespective of the disease burden [36].

Evidence have indicated that HTN severity is associated with impaired cognitive performance irrespective of the systolic and diastolic blood pressure. In fact, Muela et al. [37] have indicated that even in patients with controlled HTN (BP $<140 / 90 \mathrm{mmHg}$ under antihypertensive therapy) those in higher stage of severity had worse performance at different cognitive domains.

However, whether education could moderate the cognitive decline in hypertensive patients with high level of education is unknown. Our data suggest that hypertension might play an important role on cognitive function showing that even in patients with high level of education cognitive performance is worse when they are hypertensive. Accumulating evidence suggests that hypertension is an important risk factor for cognitive change and dementia [38]. Midlife (45-55 years of age) hypertension may be a stronger risk factor than latelife hypertension, as demonstrated in the Honolulu-Asia Aging Study midlife blood pressure [BP] was associated with dementia [39] and late-life cognitive function [40] and in a Finnish cohort for dementia and cognitive performance [41]. In the Atherosclerosis Risk in Communities (ARIC) Study, hypertension was more strongly associated with hospitalizations with a discharge code for dementia when defined in midlife vs. late life [42].

The Reykjavik study, which has been following a population from Iceland since 1967 found that the relationship between blood pressure and brain changes consistent with cognitive impairment in late life depended on whether the individual had a history of hypertension in midlife [43]. In this large population-based cohort, the authors conclude 
that late-life BP differentially affects brain pathology and cognitive performance, depending on the history of midlife hypertension. This study suggests that history of hypertension is critical to understand how late-life BP affects brain structure and function.

\section{Limitations}

The cross-sectional nature of this trial does not allow us to evaluate whether hypertension is a real predictor of cognitive dysfunction in patients whom education may play a great role on moderating the cognitive decline. Our study was carried out in a select group of patients with hypertension referred to a university hospital, limiting the generalizability of our findings to other populations.

\section{Conclusions}

In conclusion, our data suggested that hypertension impacts on cognitive impairment even in patients with high level of education. Hypertension stage, age and education were the main predictor variables of cognitive dysfunction in these patients.

\section{Competing interests}

The authors declare that they have no competing interests.

\section{Acknowledgments}

We appreciate Drs. Ricardo de Carvalho Nogueira, André Borba, Raul Feitosa, Silvia Merlim, Eduardo Sturzeneker Trés and Ana Paula Gonçalves for their valuable contributions.

\section{References}

1. Vadikolias K, Tsiakiri-Vatamidis A, Tripsianis G, Tsivgoulis G, Ioannidis P, et al. (2012). Mild cognitive impairment: Effect of education on the verbal and nonverbal tasks performance decline. Brain Behav 2:620-627. [Crossref]

2. Stern Y (2012) Cognitive reserve. Neuropsychologia 4: 2015-2028. [Crossref]

3. Neuropathology Group. Medical Research Council Cognitive Function and Aging Study (2001) Pathological correlates of late-onset dementia in a multicentre, community-based population in England and Wales. Neuropathology Group of the Medical Research Council Cognitive Function and Ageing Study (MRC CFAS). Lancet 357:169-175. [Crossref]

4. Roe CM, Xiong C, Miller JP, Morris JC (2007) Education and Alzheimer disease without dementia: Support for the cognitive reserve hypothesis. Neurology 68:223228. [Crossref]

5. Scarmeas N, Stern Y (2004) Cognitive reserve: implications for diagnosis and prevention of Alzheimer's disease. Curr Neurol Neurosci Rep 4: 374-380. [Crossref]

6. Zahodne LB, Glymour MM, Sparks C, Bontempo D, Dixon RA, et al. (2011) Education does not slow cognitive decline with aging: 12-year evidence from the victoria longitudinal study. J Int Neuropsychol Soc 17:1039-1046. [Crossref]

7. De Ronchi D, Fratiglioni L, Rucci P, Paternicò a, Graziani S, et al. (1998) The effect of education on dementia occurrence in an Italian population with middle to high socioeconomic status. Neurology 50:1231-1238. [Crossref]

8. Anstey K, Christensen H (2000) Education, activity, health, blood pressure and apolipoprotein $\mathrm{E}$ as predictors of cognitive change in old age: a review. Gerontology 46:163-177. [Crossref]

9. Bosma H, Jolles J (2003) Education and Age-Related Cognitive Decline: The contribution of mental workload. Educ Gerontol 29:165-173.

10. Butler SM, Ashford JW, Snowdon DA (1996) Age, education, and changes in the MiniMental State Exam scores of older women: findings from the Nun Study. J Am Geriatr Soc 44: 675-681. [Crossref]

11. Stern Y (2002) What is cognitive reserve? Theory and research application of the reserve concept. J Int Neuropsychol Soc 8: 448-460. [Crossref]

12. Ostrosky-Solis F, Ardila A, Rosselli M, Lopez-Arango G, Uriel-Mendoza V (1998) Neuropsychological test performance in illiterate subjects. Arch Clin Neuropsychol 13: 645-660. [Crossref]
13. Farmer ME, White LR, Abbott RD, Kittner SJ, Kaplan E, et al. (1987) Blood pressure and cognitive performance. The Framingham Study. Am J Epidemiol 126: 1103-1114. [Crossref]

14. Antelmi I, Chuang EY, Grupi CJ, Latorre M do RD de O, Mansur AJ. (2008) Heart rate recovery after treadmill electrocardiographic exercise stress test and 24-hour heart rate variability in healthy individuals. Arq Bras Cardiol 90: 380-385. [Crossref]

15. Malachias M, Plavnik FL, Machado CA, Malta D, Scala LCN, et al. (2016) 7th Brazilian Guideline of Arterial Hypertension: Chapter 1-Concept, Epidemiology and Primary Prevention Arq Bras Cardiol .107: 1-6. [Crossref]

16. Nasreddine ZS, Phillips NA, Bédirian V, Charbonneau S, Whitehead V, et al. (2005) The Montreal Cognitive Assessment, MoCA: a brief screening tool for mild cognitive impairment. J Am Geriatr Soc 53:695-699. [Crossref]

17. Torralva T, Roca M, Gleichgerrcht E, Bekinschtein T, Manes F. (2009) A neuropsychological battery to detect specific executive and social cognitive impairments in early frontotemporal dementia. Brain 132:1299-1309. [Crossref]

18. Folstein MF, Folstein SE, McHugh PR (1975) "Mini-mental state". A practical method for grading the cognitive state of patients for the clinician. J Psychiatr Res 12:189-198. [Crossref]

19. Brucki SM1, Nitrini R, Caramelli P, Bertolucci PH, Okamoto IH (2003) [Suggestions for utilization of the mini-mental state examination in Brazill. Arq Neuropsiquiatr 61: 777-781. [Crossref]

20. Memória CM, Yassuda MS, Nakano EY, Forlenza O V (2013) Brief screening for mild cognitive impairment: validation of the Brazilian version of the Montreal cognitive assessment. Int J Geriatr Psychiatry 28:34-40. [Crossref]

21. Miotto EC, Sato J, Lucia MC, Camargo CH, Scaff M (2010) Development of an adapted version of the Boston Naming Test for Portuguese speakers. Rev Bras Psiquiatr 32: 279-282. [Crossref]

22. Rosen WG (1980) Verbal fluency in aging and dementia. J Clin Neuropsychol 2:135146. [Crossref]

23. Strauss EH, Sherman EMS, Spreen O (1998) A compendium of neuropsychological tests: Adminsitration, norms, and commentary $\left(2^{\text {nd }} \mathrm{edn}\right)$. Administration Norms And Commentary: pp 1216.

24. Tombaugh TN1, Kozak J, Rees L (1999) Normative data stratified by age and education for two measures of verbal fluency: FAS and animal naming. Arch Clin Neuropsychol 14: 167-177. [Crossref]

25. Malloy-Diniz LF1, Lasmar VA, Gazinelli Lde S, Fuentes D, Salgado JV (2007) The Rey Auditory-Verbal Learning Test: applicability for the Brazilian elderly population. Rev Bras Psiquiatr 29: 324-329. [Crossref]

26. Shin MS1, Park SY, Park SR, Seol SH, Kwon JS (2006) Clinical and empirical applications of the Rey-Osterrieth Complex Figure Test. Nat Protoc 1: 892-899. [Crossref]

27. Aprahamian I, Martinelli JE, Neri AL, Yassuda MS (2010) The accuracy of the Clock Drawing Test compared to that of standard screening tests for Alzheimer's disease: results from a study of Brazilian elderly with heterogeneous educational backgrounds. Int Psychogeriatr 22:64-71. [Crossref]

28. Axelrod BN (2001) Administration duration for the Wechsler Adult Intelligence ScaleIII and Wechsler Memory Scale-III. Arch Clin Neuropsychol 16: 293-301. [Crossref]

29. Leung JL, Lee GT, Lam YH, Chan RC, Wu JY (2011) The use of the Digit Span Test in screening for cognitive impairment in acute medical inpatients. Int Psychogeriatr 23: 1569-1574. [Crossref]

30. Zahodne LB, Stern Y, Manly JJ (2015) Differing effects of education on cognitive decline in diverse elders with low versus high educational attainment. Neuropsychology 29:649-657. [Crossref]

31. Lenehan ME, Summers MJ, Saunders NL, Summers JJ, Vickers JC (2015) Relationship between education and age-related cognitive decline: a review of recent research Psychogeriatrics 15:154-162. [Crossref]

32. Dufouil C, Alpérovitch A, Tzourio C (2003) Influence of education on the relationship between white matter lesions and cognition. Neurology 60: 831-836. [Crossref]

33. Jones RN, Manly J, Glymour MM, Rentz DM, Jefferson AL, et al. (2011) Conceptual and measurement challenges in research on cognitive reserve. J Int Neuropsychol Soc 17: 593-601. [Crossref]

34. Valenzuela MJ, Sachdev P (2006) Brain reserve and dementia: a systematic review. Psychol Med 36: 441-454. [Crossref] 
35. EClipSE Collaborative Members, Brayne C, Ince PG, Keage HAD, McKeith IG, et al. (2010) Education, the brain and dementia: Neuroprotection or compensation? Brain 133: 2210-2216. [Crossref]

36. Muela HC, Costa-Hong VA, Yassuda MS, Moraes NC, et al. (2017) Hypertension Severity Is Associated With Impaired Cognitive Performance. J Am Heart Assoc 6. [Crossref]

37. Gottesman RF, Schneider AL, Albert M, Alonso A, Bandeen-Roche K, et al. (2014) Midlife hypertension and 20-year cognitive change: the atherosclerosis risk in communities neurocognitive study. JAMA Neurol 71: 1218-1227. [Crossref]

38. Freitag MH, Peila R, Masaki K, Petrovitch H, Ross GW, et al. (2006) Midlife pulse pressure and incidence of dementia: the Honolulu-Asia Aging Study. Stroke 37: 33-37. [Crossref]

39. Launer LJ, Masaki K, Petrovitch H, Foley D, Havlik RJ (1995) The association between midlife blood pressure levels and late-life cognitive function. The HonoluluAsia Aging Study. JAMA 274:1846-1851. [Crossref]
40. Kivipelto M, Helkala E, Laakso MP, Hänninen T, Hallikainen M, et al. (2001) Midlife vascular risk factors and Alzheimer's Disease in later life: Longitudinal, population based study. BMJ 322:1447-1451. [Crossref]

41. Kivipelto M, Helkala EL, Hänninen T, Laakso MP, Hallikainen M, et al. (2001) Midlife vascular risk factors and late-life mild cognitive impairment: A population-based study. Neurology 56: 1683-1689. [Crossref]

42. Alonso A, Mosley Jr. TH, Gottesman RF, Catellier D, Sharrett AR, et al. (2009) Risk of dementia hospitalisation associated with cardiovascular risk factors in midlife and older age: the Atherosclerosis Risk in Communities (ARIC) study. J Neurol Neurosurg Psychiatry 80:1194-1201. [Crossref]

43. Muller M, Sigurdsson S, Kjartansson O, Aspelund T, Lopez OL, et al. (2014) Joint effect of mid- and late-life blood pressure on the brain: the AGES-Reykjavik study. Neurology 82: 2187-2195. [Crossref]

Copyright: $@ 2017$ Muela HCS. This is an open-access article distributed under the terms of the Creative Commons Attribution License, which permits unrestricted use, distribution, and reproduction in any medium, provided the original author and source are credited. 